\title{
An outbreak of gastroenteritis associated with a novel Gll.8 sapovirus variant- transmitted by vomit in Shenzhen, China, 2019
}

Yuxiao Yan ${ }^{1,2+}$, Yuan $\mathrm{Li}^{3 \dagger}$, Wen Shi ${ }^{4+}$, Xiangyu Kong ${ }^{2}$, Huiying Li ${ }^{2}$, Qing Zhang ${ }^{2}$, Lili Pang ${ }^{2}$, Li Jiang ${ }^{5}$, Junying Liu', Miao $\operatorname{Jin}^{2^{*}} \mathrm{D}$, Yuning $\mathrm{Li}^{1,5^{*}}$ and Zhaojun Duan ${ }^{2}$

\begin{abstract}
Background: Human Sapoviruses (SaVs) has been reported as one of the causative agents of acute gastroenteritis (AGE) worldwide. An outbreak of SaVs affected 482 primary school students during spring activities from February 24 to March 11, 2019 in Shenzhen City, China. Our study was aimed at determining the epidemiology of the outbreak, investigating its origins, and making a clear identification of the SaVs genetic diversity.

Methods: Epidemiological investigation was conducted for this AGE outbreak. Stool samples were collected for laboratory tests of causative agents. Real-time reverse-transcription polymerase chain reaction (rRT-PCR) and conventional RT-PCR were used for detecting and genotyping of SaVs. The nearly complete genome of Gll.8 SaV strains were amplified and sequenced by using several primer sets designed in this study. Phylogenetic analysis was performed to characterize the genome of Gll.8 SaV strains.

Results: The single factor analysis showed that the students who were less than $1.5 \mathrm{~m}$ away from the vomitus in classroom or playgroundwere susceptible $(P<0.05)$. Seven of 11 fecal samples from patients were positive for Gll.8 SaV genotype. In this study, we obtained the genome sequence of a SaV Gll.8 strain Hu/SaV/2019008Shenzhen/2019 /CHN (SZ08) and comprehensively analyzed the genetic diversity. The phylogenetic analysis showed that the GII.8 strain SZ08 formed an independent branch and became a novel variant of Gll.8 genotype. Strain SZ08 harbored 11 specific amino acid variations compared with cluster A-D in full-length VP1.

(Continued on next page)
\end{abstract}

\footnotetext{
*Correspondence: jinmiao37@126.com; tiger19621962@163.com

${ }^{\dagger}$ Yuxiao Yan, Yuan Li and Wen Shi contributed equally to this work.

²Department of Viral Diarrhea, NHC Key Laboratory of Medical Virology and

Viral Diseases, National Institute for Viral Disease Control and Prevention,

Chinese Center for Disease Control and Prevention, 155 Changbai Rd,

Chang-ping District, Beijing 102206, China

${ }^{1}$ The First School of Clinical Medicine of Lanzhou University, Lanzhou 730000, Gansu, China

Full list of author information is available at the end of the article
}

(C) The Author(s). 2020 Open Access This article is licensed under a Creative Commons Attribution 4.0 International License, which permits use, sharing, adaptation, distribution and reproduction in any medium or format, as long as you give appropriate credit to the original author(s) and the source, provide a link to the Creative Commons licence, and indicate if changes were made. The images or other third party material in this article are included in the article's Creative Commons licence, unless indicated otherwise in a credit line to the material. If material is not included in the article's Creative Commons licence and your intended use is not permitted by statutory regulation or exceeds the permitted use, you will need to obtain permission directly from the copyright holder. To view a copy of this licence, visit http://creativecommons.org/licenses/by/4.0/. The Creative Commons Public Domain Dedication waiver (http://creativecommons.org/publicdomain/zero/1.0/) applies to the data made available in this article, unless otherwise stated in a credit line to the data. 
(Continued from previous page)

Conclusions: This study identified SaVs as the causative agents for the AGE outbreak. Strain Hu SZ08 was clustered as independent branch and there was no recombination occurred in this strain SZ08. Further, it might become the predominant strain in diarrhea cases in the near future. Constant surveillance is required to monitor the emerging variants which will improve our knowledge of the evolution of SaVs among humans.

Keywords: Sapoviruses, Outbreak, Vomit, Genome, Phylogenetic analysis

\section{Background}

There has been increasing concern about Sapoviruses (SaVs), a member of the family Caliciviridae, as a causative agent of gastroenteritis in humans in both sporadic cases and outbreaks worldwide, especially among infants and young children [1-5]. SaVs are recognized as the second most commonly etiological virus behind Norovirus in children with acute diarrhea after the successful deployment of the Rotavirus vaccine [6]. SaVs-associated outbreaks have occured in semi-closed institutions such as kindergardens, schools, hospitals and nursing homes for the elderly, and happen through the fecal-oral route, the primary route of transmission. SaVs transmit via exposure to SaVs-positive aerosols from feces, vomitus or the consumption of SaVs-contaminated food and water [7, 8]. SaVs-associated acute gastroenteritis (AGE) has increased and has been accepted as a major public health problem worldwide, especially in developing countries $[6,9,10]$.

The genome of SaVs consists of a single-stranded positive-sense RNA genome of approximately 7.1-7.5 kb in size and has a 3 '-end poly (A) tail [7]. The SaVs genome contains either 2 or 3 open reading frames (ORFs). ORF1 encodes nonstructural proteins and a major capsid protein (VP1). ORF2 encodes a minor structural protein (VP2). ORF3 predicted for several SaVs strains encodes proteins with unknown function [2, 7, 9].

Based on complete capsid gene (VP1) sequences, SaVs are classified into at least 15 genogroups (GI-GXV), four of which (GI, GII, GIV and GV) are known to infect humans. The human SaVs in genogroups GI, GII, GIV and GV are currently subdivided into 7 GI (GI.1-GI.7), 8 GII (GII.1-GII.8) and one GII.NA, 1 GIV (GIV.1) and 2 GV (GV.1 and GV.2) genotypes [11], and one additional genotype of GII.NA that has been reported recently [12].

Recently, a novel genotype of SaVs, GII.8 strains were reported in some countries and one GII.8 SaV strain (GZ2014-L231) was detected in 2014 in Guangzhou City, Guangdong province of China [13]. In this study, we reported an AGE caused by a novel GII.8 SaV variant occurred in primary school in Shenzhen City, Guangdong province of China in 2019. The genome of the GII.8 strain was analyzed and the epidemiology of this outbreak was described. The emerging of novel GII.8 $\mathrm{SaV}$ variant made a warning for SaVs outbreak control and prevention.

\section{Methods}

\section{Epidemiological investigation}

Cases were defined as at least three bouts of unformed (loose and watery) stool and/or vomiting episodes in a 24-h period during the outbreaks. A standardized questionnaire was prepared to collect demographic data (gender and age), and illness onset data (symptoms and duration of symptom).

\section{Nucleic acid extraction}

A 0.1-g fecal specimens was diluted with $1.0 \mathrm{ml}$ phosphate-buffered saline ( $\mathrm{pH} 7.2)$ to a $10 \%$ suspension. The supernatants were collected and the viral genomes were extracted by using the QIAamp Viral RNA Mini Kit (Qiagen, Hilden, Germany) and the QIAamp DNA Mini Kit (Qiagen), according to the manufacturer's instructions.

\section{Bacteria isolation}

The stool samples were analyzed for major bacterial pathogens (Escherichia coli, Salmonella, Shigella, Campylobacter and Yersinia enterocolitica) with MacConkey agar (MAC, Oxoid Ltd., Basingstoke, UK), Salmonella Shigella agar (Oxoid Ltd), Campylobacter-selective agar (Oxoid Ltd) and Yersinia Selective agar (Schiemann's CIN agar, Oxoid Ltd), respectively.

\section{Virus detection}

TaqMan real-time reverse-transcription polymerase chain reaction (rRT-PCR) was performed to test all viral nucleic acid for Norovirus and SaVs with primers and probes as described previously $[14,15]$. By using enzyme immunoassay Kits and rRT-PCR, Rotavirus A was identified [16]; and Astrovirus was identified using rRT-PCR [17]; rRT-PCR was performed to detect Adenovirus [18]. Conventional RT-PCR was performed using primer sets including P289 and P290 (in polymerase region) and SLV5317 and SLV5749 (in capsid region) to further characterize positive samples for $\mathrm{SaVs}$ as described previously [19]. The PCR cycling conditions were as follows: $42{ }^{\circ} \mathrm{C}$ for $30 \mathrm{~min}, 95^{\circ} \mathrm{C}$ for $15 \mathrm{~min}$ followed by 40 cycles of $95^{\circ} \mathrm{C}$ for $1 \mathrm{~min}, 50^{\circ} \mathrm{C}$ for $1 \mathrm{~min}$, and $72^{\circ} \mathrm{C}$ for $1 \mathrm{~min}$, and a final 10 -min elongation at $72{ }^{\circ} \mathrm{C}$. The temperature was held at $4{ }^{\circ} \mathrm{C}$ until use. 


\section{Nearly complete genome amplification}

The nearly complete genome of GII.8 strains were amplified by using 14 primer sets (Table 1) which were designed based on the whole genome of the reference strains MG674584 and MF462287. The primer set of SLV5317 and $\mathrm{VN}_{3} \mathrm{~T}_{20}$ [19] was used to amplify the complete capsid and ORF2 sequences. PCR products were sequenced using an ABI3730XL DNA Analyzer (Applied Biosystems, Foster City, CA).

\section{Genotype and sequence analysis}

These nucleotide sequences were first verified using Geneious 10.1.3 (www.geneious.com). Sequences were assembled by DNA Star software. Phylogenetic analysis of these sequences was performed in the MEGA6.0 software package (http://www.megasoftware.net/). Sequence
Alignment was performed using Clustal W. Best substitution models for the dataset were chosen based on the lowest Bayesian Information Criterion score. Phylogenetic trees with bootstrap analysis from 1000 replicates were generated by using the neighbor-joining method. SimPlot software was used to identify potential recombination among known genotypes of SaVs. DNAMAN software was used to compare the nucleotide and the amino acid identity of strain $\mathrm{Hu} / \mathrm{SaV} / 2019008$ Shenzhen/ $2019 / C H N$ (SZ08) with reference strains.

\section{Data analysis}

Statistical analysis was performed with the IBM SPSS Statistics software (Version 26.0 International Business Machines Corporation, Armonk, New York, United State). Odds ratio (OR) and 95\% confidence interval (CI)

Table 1 Primers used in this study for sapovirus detection and amplification of almost whole genome

\begin{tabular}{|c|c|c|c|c|}
\hline & Primer & Nucleotide sequence $\left(5^{\prime}-3^{\prime}\right)$ & Position & Source \\
\hline \multirow[t]{28}{*}{ Amplification of almost complete genome } & orf-1 & GAGTTGAACACGCAGTCC & $42-59^{a}$ & This study \\
\hline & & ATGATGGCACCAGTAAGG & $1009-1026^{a}$ & \\
\hline & orf-2 & CGCAGTGTCAGTGGTGTC & $880-897^{a}$ & \\
\hline & & AAGGTATGGTCGAACGAA & $1880-1897^{\mathrm{a}}$ & \\
\hline & orf-3 & CGTCGTGTTCTAACTGTTGA & $1763-1782^{a}$ & \\
\hline & & GAATCTTGTGATAACCTCCATA & $2740-2761^{a}$ & \\
\hline & orf-4 & CCAATTAGTAGCTGAAACCCTT & $2719-2740^{a}$ & \\
\hline & & CCCTTCCACGCAAACACG & $3634-3651^{a}$ & \\
\hline & orf-5 & CAGAGGGCACTTATGAGAC & $3432-3450^{\mathrm{a}}$ & \\
\hline & & CAGAGGCAGTTATGGGAG & $4434-4451^{a}$ & \\
\hline & orf-6 & GGGTCGTGTATTGTTTGG & $4335-4352^{a}$ & \\
\hline & & CCGAGTTTGGCATTTCTA & $5280-5297^{a}$ & \\
\hline & orf-7 & TAGTGTTTGAAATGGAGGGC & $5157-5176^{\mathrm{a}}$ & \\
\hline & & TTGGGAAGTGACTGCTGA & $6164-6181^{a}$ & \\
\hline & orf-8 & AGGGCATCATCTTTCCAC & $6114-6131^{a}$ & \\
\hline & & TTTGCGAGACAGTCCATTA & $7029-7047^{\mathrm{a}}$ & \\
\hline & orf-9 & GGAATCAACTGCGGAAAT & $6577-6594^{\mathrm{a}}$ & \\
\hline & & GAATAAGACAGCGATGGTC & $7429-7447^{a}$ & \\
\hline & AP1 & CTCGCCACCTATGAAGCA & $5081-5098^{a}$ & \\
\hline & & AATCGGCAGTGATGTCCT & $7004-7021^{a}$ & \\
\hline & Orf1-1 & СTTCTAAGCCATTCTACTCAA & $5-25^{b}$ & \\
\hline & & CGTGCCATGAACTGTTTG & $1179-1196^{b}$ & \\
\hline & Orf1-2 & GGCAGCATCACATCAGTC & $1093-1110^{\mathrm{b}}$ & \\
\hline & & TGTTTGCAGACATGAACG & $2078-2095^{b}$ & \\
\hline & Orf1-1,2 & СTTCTAAGCCATTCTACTCAA & $5-25^{b}$ & \\
\hline & & GCTCTGTITGCAGACATG & $6206-6323^{b}$ & \\
\hline & Orf1-4 & ACCGTGGGTGGTATGACT & $2479-2496^{b}$ & \\
\hline & & GGCGACAACCGTTGAAAT & $3269-3286^{b}$ & \\
\hline
\end{tabular}


of categorical variables were calculated using two tailed, and the comparison of categorical variables among different groups are conducted with Chi-square or Fisher's exact test. Quantitative variable was described as mean, median, standard deviation or inter-quartile range (IQR). Quantitative variable was compared by rank-sum test, analysis of variance or $\mathrm{t}$ test. Significant difference was considered as the level of $P<0.05$ with two-tailed test.

\section{Nucleotide sequences and accession number}

The $\mathrm{SaV}$ sequences determined in this study were deposited in GenBank under the accession number MT561022 for the nearly full genome sequences.

\section{Results}

\section{Epidemiological investigation}

This AGE outbreak occurred in a primary school in Shenzhen City in China. It started on Feb 24th and lasted until March 11th 2019. It included 482 cases among the 1850 students and 95 teachers of the school. The school has 34 classes divided into 6 different grades. There were two buildings in which students of grade 3 and 4 were in Building $A$ and students of grade 5-6 were in Building B. The students from grade 4-6 were organized to participate in military training activities during February 26-27 while the students from grade 1-3 were organized to participate in spring outing on February 27 after the school opened on February 25. The age of 482 cases ranged from 9 to 14 years. The first case came from class 4 , grade 5 , and had symptoms one day (February 24th) before school began after winter vacation (February 25th). He did not rest at home, still returned to school on school day and vomited in classroom. During the subsequent military training activities, he vomited on the bus (February 26th). The students of grade 5 got sick first and then grade 4,6 and 3 . There was no case from grade 1 and 2. Peak of cases occurred on March 1 on which local district CDC was invited to investigate this outbreak and the second peak of cases occurred on March 4 on which classes of G4-6 were suspended and were resumed on March 8. Classes of G3 were suspended on March 8 and resumed on March 11 (Fig. 1). The characteristic symptoms within the cohort were diarrhea $(67 \%$, $321 / 482)$, vomiting $(45 \%, 218 / 482)$, dizziness $(30 \%$, $144 / 482)$, stomach pain $(22 \%, 106 / 482)$ and nausea $(18 \%, 86 / 482)$. The results showed that the attack rate of each grade was $36 \%$ in Grade $5,23 \%$ in Grade 6 , $21 \%$ in grade 4 and $6 \%$ in grade 3 , respectively $(P<$ 0.05). The attack rate of case each floor of Building A was $3 \%$ for the 4 th floor, $20 \%$ for the 5 th floor $\left(x^{2}=\right.$ $45.09, P<0.01)$, and the attack rate of case each floor of Building B was $41 \%$ for the 3rd floor, $38 \%$ for the

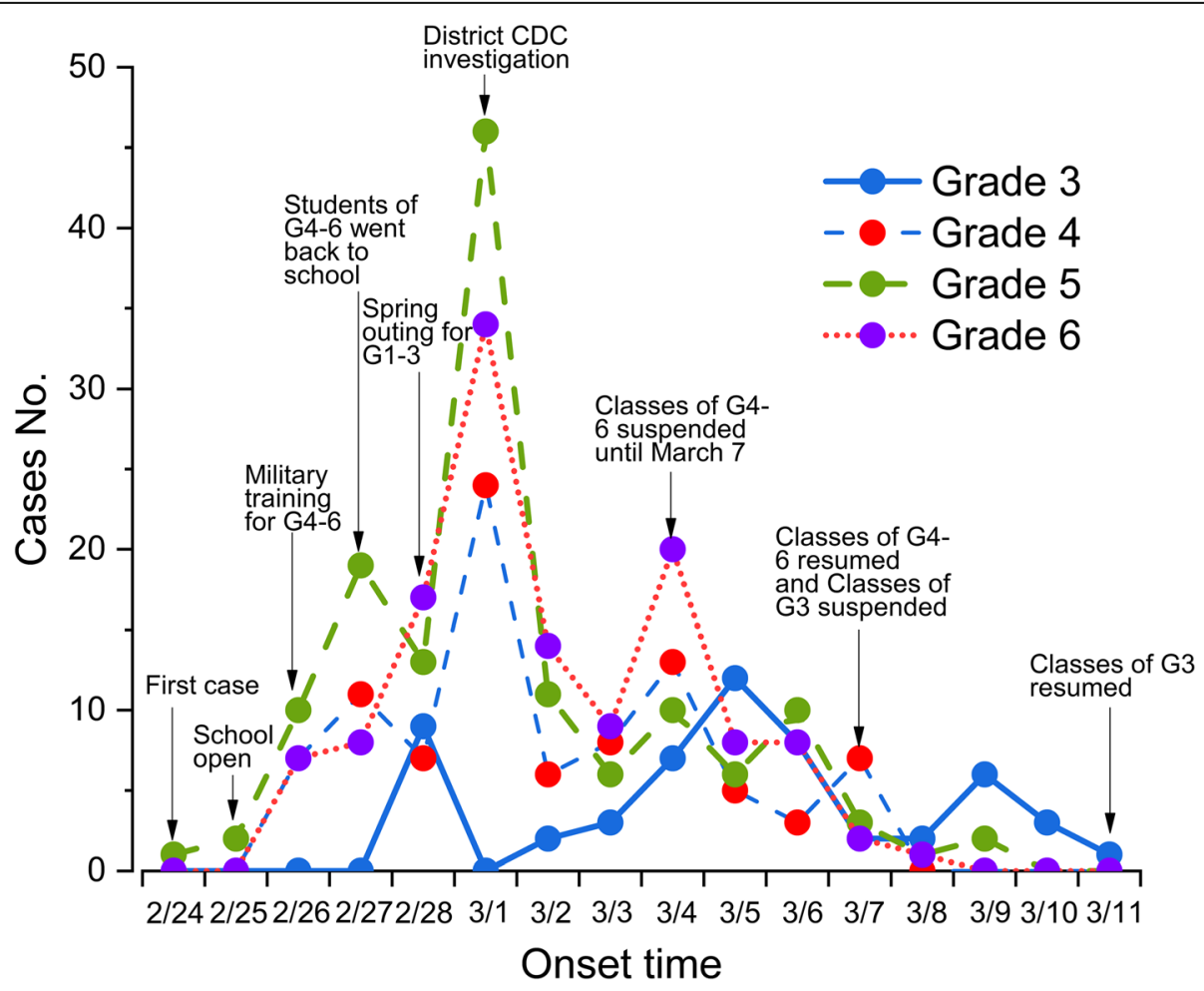

Fig. 1 Epidemic curve of acute gastroenteritis cases in the outbreak occurred in Shenzhen, China, 2019 
4th floor, and $20 \%$ for the 2th floor $\left(x^{2}=16.44, P<\right.$ $0.05)$. The difference of case incidence among different floors was statistically significant (Fig. 2a, b).

During the epidemic, 218 students vomited. Vomiting occurred in plastic bags (13\%), floor (10\%) and window (1\%) while riding the bus and vomiting occurred in the bathroom (3\%) and dormitory (2\%) during activity. Vomiting occurred in Classrooms (9\%), playgrounds (3\%), hallway (3\%) after returning to school (Table 2). In the group with common exposure to vomit, the single factor analysis showed that among the students that saw other peers vomiting in the classroom or playground, being at a distance of $1.5 \mathrm{~m}$ or less of the vomiting event which provided more chances to access to the virus, make them more susceptible to the infection $(P<0.05)$ (Table 3).

\section{Pathogen investigation}

A total of these pathogens, including E. coli Salmonella, Shigella, Campylobacter, Yersinia enterocolitica, Norovirus, Rotavirus, Adenovirus and Astrovirus were detected to be negative. Sequence analysis of several positive rRT-PCR samples revealed identical sequences, which were identified as SaVs. SaVs were detected in 7 of 11 stool specimens collected from the cases of the outbreak .

\section{Analysis of nearly complete genome sequence}

The nearly complete genomic sequence of strain SZ08 was obtained and its length was $7338 \mathrm{nt}$ excluding the sequences of 5 'UTRs and the 3 '-end poly (A) tail in this study. The sequenced genomes were predicted to contain two major ORFs from 1 to 6837 (ORF1, encoding the nonstructural proteins and major capsid protein VP1) and from 6838 to 7337 (ORF2, encoding the minor structural protein). Its 3 '-UTR had $52 \mathrm{nt}$. The reference strain MF462288/ GII.8/Peru330/PNV010961/2008 shared the highest query cover and nucleotide identities (93.8\%) with the strain SZ08 based on nearly complete genome. The nucleotide identities of the strain SZ08 with strain Peru330 were 93.13\% in the ORF1, 94.61\% in ORF2 and $93.30 \%$ in ORF3, respectively. According to the amino acid identities of the protein alignment, a great degree of similarity $(96.95 \%$ at the nearly complete

\section{A}
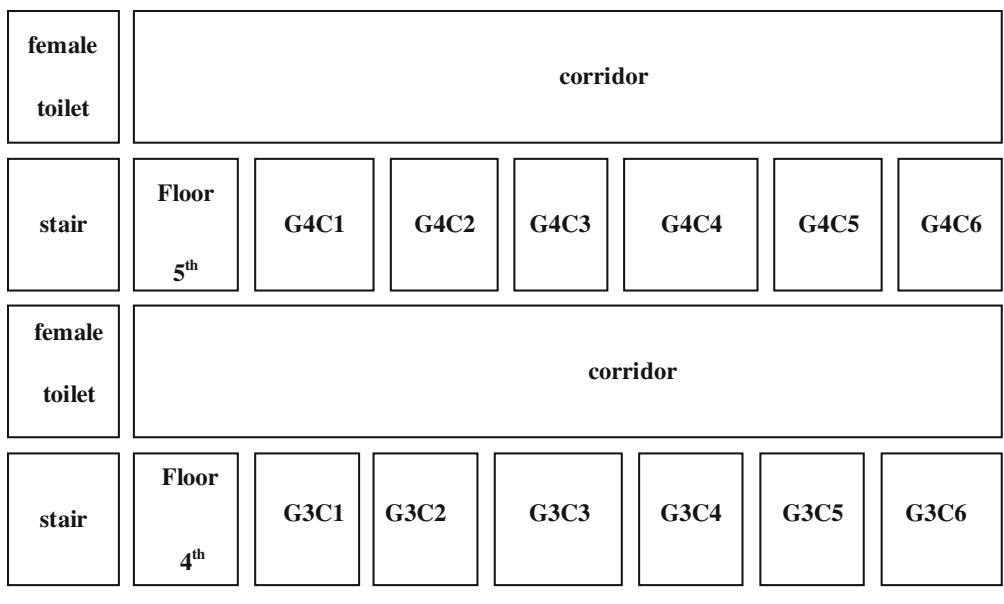

corridor

male

toilet

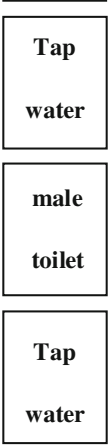

B

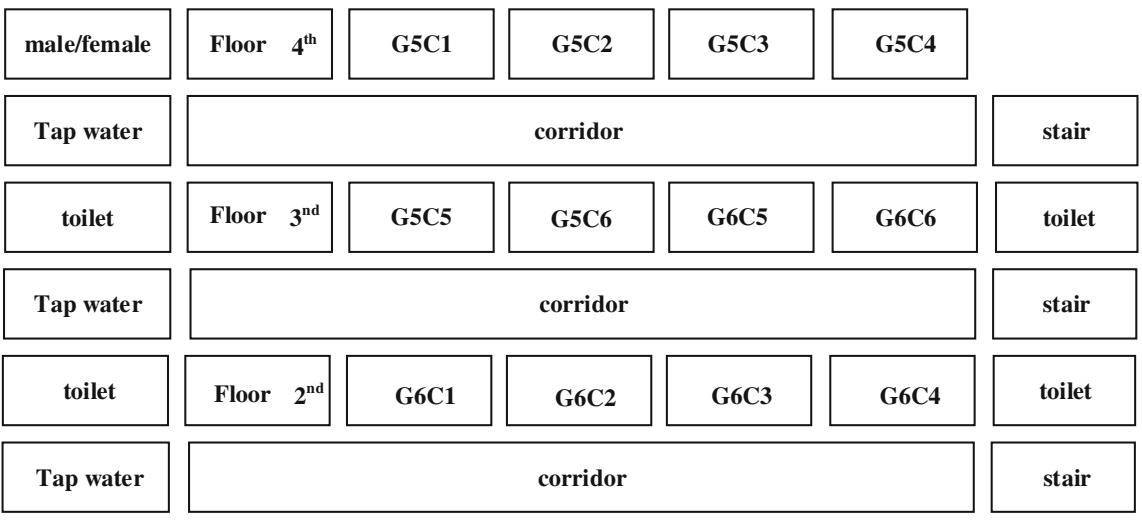

Fig. 2 a Schematic diagram of each floor of BuildingA. b Schematic diagram of each floor of BuildingB 
Table 2 Distribution of vomiting places of students

\begin{tabular}{lll}
\hline Place of vomiting & No. of vomiting cases & $\%$ \\
\hline Bus & 28 & 13 \\
Plastic bag & 10 & 5 \\
Ground & 2 & 1 \\
Outside the window & & \\
Military training & 6 & 3 \\
Bathroom & 4 & 2 \\
Dorm & 4 & 2 \\
Playground & 1 & 0 \\
Canteen & & \\
School & 61 & 28 \\
Toilet & 20 & 9 \\
Classroom & 7 & 3 \\
Playground & 6 & 3 \\
Doorway & 13 & 6 \\
Other & &
\end{tabular}

genome, $98.46 \%$ in the nonstructural proteins and VP1 and $97.54 \%$ in the VP2) were shown between them. We observed 48 aa variations in both nonstructural proteins and VP1 and 25 aa variations in the minor structural protein.

\section{Analysis of complete capsid protein}

According to the phylogenetic analysis, Strain SZ08 clustered into GII.8 branch and was independent with other clusters (A, B, C and D) named previously [13] and was named after cluster $\mathrm{E}$ (Fig. 3). The nucleotide identity of the strain SZ08 was ranged from 92.94 to $94.02 \%$ with cluster A-D and the amino acid identity was ranged from 96.76 to $97.48 \%$. However, cluster A-D shared the high nucleotide identity (96.87-99.84\%) and amino acid identity (98.31-99.88\%). Strain SZ08 harbored 11 specific amino acid variations (S249A, A275S, S299A,
A301T, G302T, V335N, L465I, S497N, I503V, A528V and V552I) compared with cluster A-D. However, five original amino acids had the transversion (S43T, S183A, E341D, T403S, I520V) from the earliest strain Peru330/ PNV010961/2008 (cluster A). Cluster B-D had low amino acid variations (Fig. 4).

\section{Discussion}

The epidemiology of cases of AGE of viral etiology is a relevant public health issue [20]. Of note, reports on SaVs-associated AGE across all age groups have recently increased worldwide $[6,13,21]$, which indicated that the cases caused by SaVs may become extensive and global. And Shenzhen is also one of the high-incidence areas of SaVs-associated outbreaks recently in mainland China $[19,22]$. Human SaVs cause AGE in all age groups in both sporadic cases and outbreaks worldwide as well as predominantly occurs in children [23, 24], which correspond to this study. On the other hand, the outbreak described by the study occured in primary school and expose to vomit was recognized as the cause, which are similar to the previous studies [25, 26].

The investigation showed the difficulty to prove the person-to-person transmission by contact or from aerosols generated by vomit [27-29]. In this study, we investigated the vomiting places and the single factor analysis showed that the participation in the handling of vomitus and the distance of vomitus were the main factors causing gastroenteritis infection (logistic regression, $P<0.05$ ). It has been proposed that the formation of aerosols that can remain in the air for some time and can then be breathed in and swallowed or through contamination of surface causes the outbreaks [30-32]. The ingestion of aerosolized vomitus, aerosols transmission via vomiting might explain the partial gastroenteritis outbreaks which are not caused by foodborne or waterborne outbreaks as described previously [32-35]. Vomit was considered only when exposure was at $<1 \mathrm{~m}$, however, possible

Table 3 Case control study on risk factors of acute gastroenteritis outbreak caused by Sapovirus

\begin{tabular}{|c|c|c|c|c|c|c|c|c|c|}
\hline \multirow[t]{2}{*}{ No. } & \multicolumn{2}{|l|}{ Case } & \multicolumn{2}{|c|}{ Non case } & \multirow{2}{*}{$\begin{array}{l}\text { OR } \\
\text { value }\end{array}$} & \multicolumn{2}{|l|}{$95 \% \mathrm{Cl}$} & \multirow[t]{2}{*}{$x^{2}$} & \multirow{2}{*}{$\begin{array}{l}P \\
\text { value }\end{array}$} \\
\hline & Expose & $\%$ & Expose & $\%$ & & lower limit & Upper limit & & \\
\hline See others vomiting & 278 & 58 & 365 & 46 & 1.58 & 1.26 & 1.99 & 15.6 & 0.00 \\
\hline See others vomiting in the classroom & 120 & 25 & 138 & 17 & 1.56 & 1.19 & 2.06 & 10.14 & 0.00 \\
\hline See others vomiting in the playground & 36 & 7 & 35 & 4 & 1.74 & 1.08 & 2.81 & 5.22 & 0.02 \\
\hline Less than $1.5 \mathrm{~m}$ from vomit & 156 & 32 & 166 & 21 & 1.42 & 1.04 & 1.95 & 4.81 & 0.03 \\
\hline Handling vomitus & 45 & 9 & 36 & 5 & 2.17 & 1.37 & 3.41 & 11.57 & 0.00 \\
\hline See others Vomiting on bus & 86 & 18 & 116 & 15 & 1.25 & 0.92 & 1.7 & 2.11 & 0.15 \\
\hline See others Vomiting during activity & 14 & 3 & 30 & 4 & 0.76 & 0.4 & 1.44 & 0.72 & 0.40 \\
\hline See others Vomiting in bathroom & 56 & 12 & 71 & 9 & 1.33 & 0.92 & 1.93 & 2.28 & 0.13 \\
\hline Self protection in handling & 12 & 27 & 7 & 19 & 1.93 & 0.69 & 5.4 & 1.62 & 0.20 \\
\hline Family members in the same school & 116 & 24 & 170 & 22 & 1.15 & 0.88 & 1.51 & 1.06 & 0.30 \\
\hline
\end{tabular}




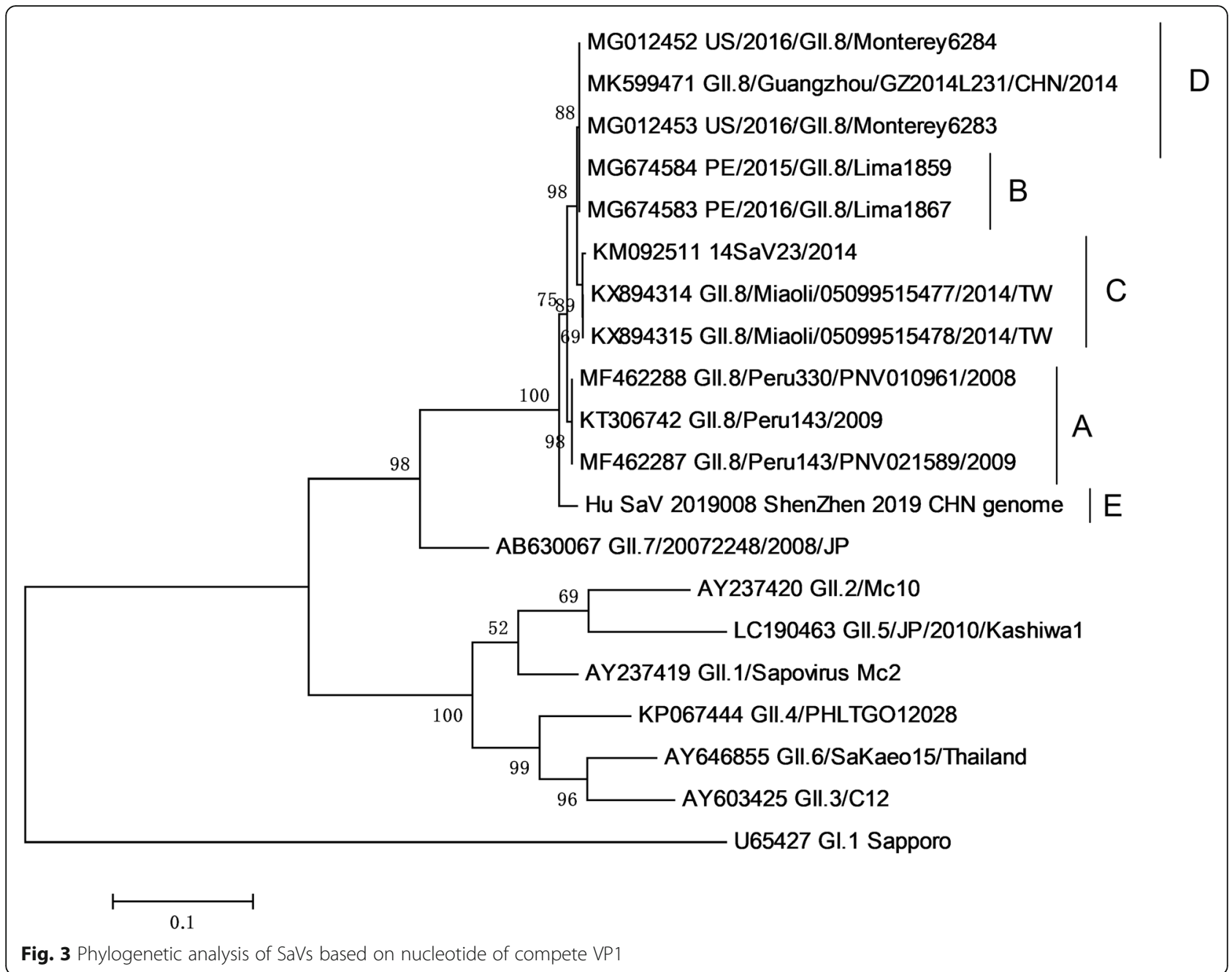

exposures at greater distances have also been reported $[27,28]$. Our study showed the distance under $1.5 \mathrm{~m}$ could cause the infection. Therefore, the study gave the evidence that the greater distance also could cause the cases. Since vomiting is a common symptom in calicivirus outbreaks, ingestion of aerosolized vomitus, may exist in most $\mathrm{SaVs}$ outbreaks. Therefore, ingestion of aerosolized vomitus, should be valued in the investigation of gastroenteritis outbreaks [28].

Emerging virus strains often have a risk of causing a pandemic. SaV GII.8 was first identified in two hospitalized children's samples in Peru, in 2008 [36]. In mainland China, GII.8 was first reported in Shenzhen, in 2011 [22]. Additionally, in 2019, Xue al. acquired the first GII.8 SaV genome from mainland China [13]. Based on the phylogenetic analysis, the strain SZ08 in this study was classified as a member of GII.8 SaV. In addition, strain SZ08 isolated in 2019 was clustered as independent branch and was different from the strain GZ2014-L231 isolated in 2014 although strain of Shenzhen and strain GZ2014-L231 of Guangzhou were both from the same province, Guangdong. GII.8 strains were also detected in other countries not only in clinical but also in environmental samples $[37,38]$. SaVs outbreak

\begin{tabular}{|c|c|c|c|c|c|c|c|c|c|c|c|c|c|c|c|c|c|c|c|c|c|}
\hline Cluster & name & 4 & 10 & $\begin{array}{ll}7 & 1\end{array}$ & 32 & 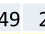 & 27529 & 629 & 930 & 130 & 1233 & & 4140 & $03 \quad 44$ & & $465 \quad 4$ & 97 & 503 & 520 & & 552 \\
\hline A & MF462288_GII.8/Peru330/PNV010961/2008 & S & Y & $A$ & $S$ & A & $\mathrm{F}$ & $\mathrm{S}$ & A & G & V & D & S & M & $\mathrm{L}$ & S & I & V & A & V & $\mathrm{V}$ \\
\hline c & KX894314_GII.8/Miaoli/05099515477/2014/TW & $\mathrm{T}$ & $\mathrm{F}$ & $\mathrm{S}$ & $\mathrm{S}$ & A & Y & $\mathrm{s}$ & $\mathrm{s}$ & G & I & $\mathrm{E}$ & $\mathrm{T}$ & M & L & $\mathrm{S}$ & I & I & A & V & $\mathrm{V}$ \\
\hline D & MK599471_GII.8/Guangzhou/GZ2014L231/CHN/2014 & $S$ & $\mathrm{~F}$ & $S$ & $S$ & A & Y & $S$ & $\mathrm{~S}$ & G & V & E & $\mathrm{T}$ & M & L & S & I & I & A & V & V \\
\hline B & MG674583_PE/2016/GII.8/Lima1867 & $\mathrm{S}$ & $\mathrm{F}$ & S & $\mathrm{S}$ & A & Y & $\mathrm{S}$ & $\mathrm{S}$ & G & V & $\mathrm{E}$ & $\mathrm{T}$ & M & $\mathrm{L}$ & $\mathrm{S}$ & I & I & A & V & $\mathrm{V}$ \\
\hline E & SaV 2019008 Shenzhen 2019 CHN & $\mathrm{S}$ & $\mathrm{F}$ & A & A & S & Y & A & $\mathrm{T}$ & $\mathrm{T}$ & $\mathrm{N}$ & D & S & M & & $\mathrm{N}$ & V & V & V & & \\
\hline
\end{tabular}

Fig. 4 Amino acid variation in complete capsid protein gene compared to reference strains of SaV Gll.8 
caused by GII.8 strains occurred in long term center in previous report [36] and the GII.8 SaV outbreak occurred in primary school. It suggested the GII.8 strain could cause the children and the elderly to infect. Despite the low detection rate, the wide spread distribution of the virus in different countries and wide age groups, make it as important concern to understand its genetic diversity and evolutionary characteristics.

Recombination and mutations are the two important way of viral evolution not only in SaVs but also in Noroviruses [13,39]. According to the genomic analysis, there was no recombination occurred in strain SZ08.

Amino acid variation in protein is the important way of viral evolution. In this study, by comparing the differences between the strain SZ08 as new cluster and GII.8 human $\mathrm{SaV}$ representative strains of other clusters, more amino acid variation occurred in VP1 capsid region in which potential epitopes and receptor binding sites located. The variation in VP1 might be one of the explanations why there was the large scale of cases in this SaVs outbreak in this study.

In conclusion, contacting vomitus could be one of important transmission mechanisms which may explain a large number of cases of $\mathrm{SaVs}$ outbreaks. It is noteworthy that SaVs-associated diarrhea is generally mild, while large-scale outbreaks by $\mathrm{SaV}$ may also occur. In addition, we obtained the genome of a novel GII.8 SaV variant from China and comprehensively analyzed the genomic characteristics. The results of this study could not only provide reference data for $\mathrm{SaVs}$ researches in the future, but also deepen the understanding of evolution mechanisms of the new GII.8 variant. Constant surveillance is required to monitor the emergence of these strains and will make a clear identification of changes in major strains and improve our knowledge of the evolution of SaVs among humans.

\section{Abbreviations}

SaVs: Sapoviruses; AGE: Acute gastroenteritis; rRT-PCR: Reverse-transcription polymerase chain reaction; ORFs: Open reading frames; OR: Odds ratio; $\mathrm{Cl}$ : Confidence interval; IQR: Inter-quartile range

\section{Acknowledgments}

We thank Director Huilai Ma and deputy Director Lijie Zhang from the Chinese Field Epidemiology Training Project for their guidance to the epidemiological investigation of this sapovirus outbreak in this study. We thank the staff from Baoan Center for Disease Control and Prevention in Shenzhen for participating in this outbreak.

\section{Authors' contributions}

YY performed the study, participated in the analysis of the study and wrote the manuscript. MJ designed the study and modify the manuscript. $Y L$ and WS conducted the epidemiological investigation and collected specimen. $\mathrm{XK}, \mathrm{HL}, \mathrm{QZ}$ and LP participated in the experiment of pathogenic detection, genotyping and genome amplification. $\amalg$ and $J L$ participated in the statistical analysis. YL and ZD modified the manuscript. The author(s) read and approved the final manuscript.
Funding

We thank The Key Project of Science and Technology (Grant No. 2018ZX10301408-001 and 2018ZX10102-001) for their significant contribution to the outbreak investigation.

\section{Availability of data and materials}

The datasets used and analyzed during this study are available from the corresponding author on reasonable request.

\section{Ethics approval and consent to participate}

This work was conducted in agreement with the research Ethics committee and the Institutional Review Board at the Baoan district Center for Disease Control and Prevention for human subject projection. We informed each potential subject of the details of study and written informed consent was obtained from the parents or legal guardians of all subjects.

\section{Consent for publication}

Not applicable.

\section{Competing interests}

The authors declare that they have no competing interests.

\section{Author details}

${ }^{1}$ The First School of Clinical Medicine of Lanzhou University, Lanzhou 730000, Gansu, China. ${ }^{2}$ Department of Viral Diarrhea, NHC Key Laboratory of Medical Virology and Viral Diseases, National Institute for Viral Disease Control and Prevention, Chinese Center for Disease Control and Prevention, 155 Changbai Rd, Chang-ping District, Beijing 102206, China. ${ }^{3}$ Shenzhen Baoan Center for Disease Control and Prevention, Baoan District, Shenzhen, Guangdong Province, China. ${ }^{4}$ Yingkou Center for Disease Control and Prevention, Yingkou, Liaoning, China. ${ }^{5}$ The First Hospital of Lanzhou University, Donggang-xi Rd, Cheng-guan District, Lanzhou 730000, Gansu, China. ${ }^{6}$ Central Hospital of Zhoukou, Zhoukou 466000, Henan, China.

Received: 4 June 2020 Accepted: 22 November 2020

Published online: 01 December 2020

\section{References}

1. Cilli A, Luchs A, Leal E, et al. Human sapovirus GI.2 and GI.3 from children with acute gastroenteritis in northern Brazil. Mem Inst Oswaldo Cruz. 2019; 114:e180574. https://doi.org/10.1590/0074-02760180574.

2. Usuku S, Kumazaki M. A gastroenteritis outbreak attributed to sapovirus genogroup v in Yokohama, Japan. Jpn J Infect Dis. 2014;67(5):411-2. https:// doi.org/10.7883/yoken.67.411.

3. Luo LF, Qiao K, Wang XG, et al. Acute gastroenteritis outbreak caused by a Gll.6 norovirus. World J Gastroenterol. 2015;21(17):5295-302. https://doi.org/ 10.3748/wjg.v21.i17.5295.

4. Ike AC, Hartelt K, Oehme RM, et al. Detection and characterization of sapoviruses in outbreaks of gastroenteritis in southwest Germany. I Clin Virol. 2008;43(1):37-41. https://doi.org/10.1016/j.jcv.2008.04.003.

5. Kobayashi S, Fujiwara N, Yasui Y, et al. A foodborne outbreak of sapovirus linked to catered box lunches in Japan. Arch Virol. 2012;157(10):1995-7. https://doi.org/10.1007/s00705-012-1394-8.

6. Liu $X$, Jahuira $H$, Gilman RH, et al. Etiological role and repeated infections of sapovirus among children aged less than 2 years in a cohort study in a Periurban community of Peru. J Clin Microbiol. 2016;54(6):1598-604. https://doi. org/10.1128/jcm.03133-15.

7. Oka T, Wang Q, Katayama K, et al. Comprehensive review of human sapoviruses. Clin Microbiol Rev. 2015;28(1):32-53. https://doi.org/10.1128/ cmr.00011-14.

8. He $\mathrm{Y}$, Jin $\mathrm{M}$, Chen $\mathrm{K}$, et al. Gastroenteritis outbreaks associated with the emergence of the new Gll.4 Sydney norovirus variant during the epidemic of 2012/13 in Shenzhen city, China. PLoS One. 2016;11(11):e0165880. https://doi.org/10.1371/journal.pone.0165880.

9. Iritani N, Yamamoto SP, Abe N, et al. Epidemics of GI.2 sapovirus in gastroenteritis outbreaks during 2012-2013 in Osaka city, Japan. J Med Virol. 2016;88(7):1187-93. https://doi.org/10.1002/jmv.24451.

10. Phan TG, Trinh $Q D$, Yagyu $F$, et al. Emergence of rare sapovirus genotype among infants and children with acute gastroenteritis in Japan. Eur J Clin Microbiol Infect Dis. 2007;26(1):21-7. https://doi.org/10. 1007/s10096-006-0235-7. 
11. Kumthip K, Khamrin P, Ushijima H, et al. Genetic recombination and diversity of sapovirus in pediatric patients with acute gastroenteritis in Thailand, 2010-2018. PeerJ. 2020;8:e8520. https://doi.org/10.7717/peerj.8520.

12. Diez-Valcarce M, Montmayeur A, Tatusov R, et al. Near-complete human sapovirus genome sequences from Kenya. Microbiol Resour Announc. 2019; 8(7). https://doi.org/10.1128/mra.01602-18.

13. Xue L, Cai W, Gao J, et al. Genome characteristics and molecular evolution of the human sapovirus variant Gll.8. Infect Genet Evol. 2019;73:362-7. https://doi.org/10.1016/j.meegid.2019.05.017.

14. Kageyama T, Kojima S, Shinohara M, et al. Broadly reactive and highly sensitive assay for Norwalk-like viruses based on real-time quantitative reverse transcription-PCR. J Clin Microbiol. 2003;41(4):1548-57. https://doi. org/10.1128/jcm.41.4.1548-1557.2003.

15. Oka T, Katayama K, Hansman GS, et al. Detection of human sapovirus by real-time reverse transcription-polymerase chain reaction. J Med Virol. 2006; 78(10):1347-53. https://doi.org/10.1002/jmv.20699.

16. Magzoub MA, Bilal NE, Bilal JA, et al. Detection and sequencing of rotavirus among sudanese children. Pan Afr Med J. 2017;28:87. https://doi.org/10. 11604/pamj.2017.28.87.11008.

17. Resque HR, Munford V, Castilho JG, et al. Molecular characterization of astrovirus in stool samples from children in Sao Paulo, Brazil. Mem Inst Oswaldo Cruz. 2007;102(8):969-74. https://doi.org/10.1590/s007402762007000800012

18. Allard A, Albinsson B, Wadell G. Rapid typing of human adenoviruses by a general PCR combined with restriction endonuclease analysis. J Clin Microbiol. 2001;39(2):498-505. https://doi.org/10.1128/jcm.39.2.498-505.2001.

19. Wang Md J, Li Ph DY, Kong Md X, et al. Two gastroenteritis outbreaks caused by sapovirus in Shenzhen, China. J Med Virol. 2018;90(11):1695-702. https://doi.org/10.1002/jmv.25236.

20. Oka T, Mori K, Iritani N, et al. Human sapovirus classification based on complete capsid nucleotide sequences. Arch Virol. 2012;157(2):349-52. https://doi.org/10.1007/s00705-011-1161-2.

21. Yamashita Y, Ootsuka Y, Kondo R, et al. Molecular characterization of Sapovirus detected in a gastroenteritis outbreak at a wedding hall. J Med Virol. 2010;82(4):720-6. https://doi.org/10.1002/jmv.21646.

22. Wu W, Yang $H$, Zhang $H L$, et al. Surveillance of pathogens causing gastroenteritis and characterization of norovirus and sapovirus strains in Shenzhen, China, during 2011. Arch Virol. 2014;159(8):1995-2002. https://doi. org/10.1007/s00705-014-1986-6.

23. Svraka S, Vennema H, Van Der Veer B, et al. Epidemiology and genotype analysis of emerging sapovirus-associated infections across Europe. J Clin Microbiol. 2010;48(6):2191-8. https://doi.org/10.1128/jcm.02427-09.

24. Phan TG, Khamrin P, Quang TD, et al. Emergence of intragenotype recombinant sapovirus in Japan. Infect Genet Evol. 2007;7(4):542-6. https:// doi.org/10.1016/j.meegid.2007.02.004

25. Yu Y, Guo XH, Yan HQ, et al. Systematic review on the characteristics of acute gastroenteritis outbreaks caused by sapovirus. Zhonghua Liu Xing Bing Xue Za Zhi. 2019;40(1):93-8. https://doi.org/10.3760/cma.j.issn.02546450.2019.01.019.

26. Becker-Dreps S, Bucardo F, Vinje J. Sapovirus: an important cause of acute gastroenteritis in children. Lancet Child Adolesc Health. 2019;3(11):758-9. https://doi.org/10.1016/s2352-4642(19)30270-6.

27. Makison Booth C. Vomiting Larry: a simulated vomiting system for assessing environmental contamination from projectile vomiting related to norovirus infection. J Infect Prev. 2014;15(5):176-80. https://doi.org/10.1177/ 1757177414545390.

28. Marks PJ, Vipond IB, Carlisle D, et al. Evidence for airborne transmission of Norwalk-like virus (NLV) in a hotel restaurant. Epidemiol Infect. 2000;124(3): 481-7. https://doi.org/10.1017/s0950268899003805.

29. Widdowson MA, Glass R, Monroe $S$, et al. Probable transmission of norovirus on an airplane. JAMA. 2005;293(15):1859-60. https://doi.org/10.1001/jama. 293.15.1859.

30. Lopman B. Air sickness: vomiting and environmental transmission of norovirus on aircraft. Clin Infect Dis. 2011;53(6):521-2. https://doi.org/10. 1093/cid/cir486

31. Kirking $\mathrm{HL}$, Cortes J, Burrer $\mathrm{S}$, et al. Likely transmission of norovirus on an airplane, October 2008. Clin Infect Dis. 2010;50(9):1216-21. https://doi.org/ 10.1086/651597.

32. Marks PJ, Vipond IB, Regan FM, et al. A school outbreak of Norwalk-like virus: evidence for airborne transmission. Epidemiol Infect. 2003;131(1):72736. https://doi.org/10.1017/s0950268803008689.
33. Godoy P, Artigues A, Bartolome R, et al. Norovirus gastroenteritis outbreak by person-to-person transmission in a nursing home. Med Clin (Barc). 2006; 127(14):538-41. https://doi.org/10.1016/s0025-7753(06)72322-3.

34. Harris JP, Lopman BA, Cooper BS, et al. Does spatial proximity drive norovirus transmission during outbreaks in hospitals? BMJ Open. 2013;3(7). https://doi.org/10.1136/bmjopen-2013-003060.

35. Wikswo ME, Cortes J, Hall AJ, et al. Disease transmission and passenger behaviors during a high morbidity Norovirus outbreak on a cruise ship, January 2009. Clin Infect Dis. 2011;52(9):1116-22. https://doi.org/10.1093/ $\mathrm{cid} / \mathrm{cir} 144$.

36. Diez-Valcarce $M$, Castro CJ, Marine RL, et al. Genetic diversity of human sapovirus across the Americas. J Clin Virol. 2018;104:65-72. https://doi.org/ 10.1016/j.jcv.2018.05.003

37. Kagning Tsinda E, Malasao R, Furuse $Y$, et al. Complete coding genome sequences of uncommon Gll.8 sapovirus strains identified in diarrhea samples collected from Peruvian children. Genome Announc. 2017;5(43). https://doi.org/10.1128/genomeA.01137-17.

38. Kitajima M, Rachmadi AT, Iker BC, et al. Temporal variations in genotype distribution of human sapoviruses and Aichi virus 1 in wastewater in southern Arizona, United States. J Appl Microbiol. 2018;124(5):1324-32. https://doi.org/10.1111/jam.13712.

39. White PA. Evolution of norovirus. Clin Microbiol Infect. 2014;20(8):741-5. https://doi.org/10.1111/1469-0691.12746.

\section{Publisher's Note}

Springer Nature remains neutral with regard to jurisdictional claims in published maps and institutional affiliations.
Ready to submit your research? Choose BMC and benefit from:

- fast, convenient online submission

- thorough peer review by experienced researchers in your field

- rapid publication on acceptance

- support for research data, including large and complex data types

- gold Open Access which fosters wider collaboration and increased citations

- maximum visibility for your research: over $100 \mathrm{M}$ website views per year

At BMC, research is always in progress.

Learn more biomedcentral.com/submissions 